() [2009] IEEE. Reprinted, with permission, from Shao. Zhongkai., Zhan, Yuedong., and Guo, Youguang. 2009, 'Fuzzy Neural Network-based Model Reference Adaptive Inverse Control for Induction Machines', Proceedings of IEEE International

Conference on Applied Superconductivity and Electromagnetic Devices, pp. 56-59. This material is posted here with permission of the IEEE. Such permission of the IEEE does not in any way imply IEEE endorsement of any of the University of Technology, Sydney's products or services. Internal or personal use of this material is permitted. However, permission to reprint/republish this material for advertising or promotional purposes or for creating new collective works for resale or redistribution must be obtained from the IEEE by writing to pubs-permissions@ieee.org. By choosing to view this document, you agree to all provisions of the copyright laws protecting it. 


\section{Fuzzy Neural Network-based Model Reference Adaptive Inverse Control for Induction Machines}

\author{
Zongkai Shao \\ School of Hydropower \& Information \\ Engineering \\ Huazhong University of Science and \\ Technology \\ Wuhan, China \\ shaozongkai@126.com
}

\author{
Yuedong Zhan \\ Department of Automation \\ Kunming University of Science and \\ Technology \\ Kunming, China \\ ydzhan@163.com
}

\author{
Jianguo Zhu, Youguang Guo \\ School of Electrical, Mechanical and \\ Mechatronic Systems \\ University of Technology \\ Sydney, Australia \\ joe@eng.uts.edu.au, \\ youguang@eng.uts.edu.au
}

\begin{abstract}
In this paper, because the induction machines are described as the plants of highly nonlinear and parameters timevarying, in order to obtain a very well control performances that a conventional model reference adaptive inverse control (MRAIC) can not be gotten, a fuzzy neural network-based model reference adaptive inverse control strategy for induction motors is presented based on the rotor field oriented motion model of induction machines. The fuzzy neural network control (FNNC) is incorporated into the model reference adaptive control (MRAC), a fuzzy basis function network controller (FBNC) and a fuzzy neural network identifier (FNNI) for asynchronous motors adjustable speed system are designed. The proposed controller for asynchronous machines resolves the shortage of MRAC, and employs the advantages of FNNC and MRAC. Simulation results show that the proposed control strategy is of the feasibility, correctness and effectiveness.
\end{abstract}

Index Terms-induction machine, machine dynamic model, fuzzy neural network control (FNNC), model reference adaptive control (MRAC)

\section{INTRODUCTION}

With the development of power electronics and computer control technology, induction motor (IM) adjustable speed systems have been widely applied in the situation of high voltage, large power, and high performance. The IM adjustable speed systems possesses many advantages, such as wide range and high accuracy of regulating speed, high stability, fast dynamic response, able to operate in four-quadrant, and etc.. Among many application examples are the coal, petroleum, chemical plant, and metallurgy industry. At present, the IM adjustable speed system is being developed towards higher performance, higher precision, larger capacity, digitalization, integration and intelligence [1].

Regarding the study of the modeling and inverse control of IM, Liu et al. [2] proposed a speed estimation based on neural network inversion, which was a multi-layer feed -forward neural network trained by advanced Back Propagation arithmetic. Li et el. [3] carried on a research on extended Kalman filter (EKF) based on inverse $\Gamma$ model, on which state noises the change of motor parameters was taken, and it made more precision for vector control. Liu et el. [4] developed a decoupling control approach based on $\alpha$-th inverse system for the innovative 5 degree-of-freedom bearingless induction motor, which was multi-variable, nonlinear and high coupling system. Dai et al. [5] proposed a neural network inverse control (NNIC) structure and gave the neural network inverse system (NNIS) of induction motor in rotor field oriented (MT) reference frame as a special case, the comparison of this NNIC with direct rotor field oriented control (DRFOC) was done.

This paper firstly analyses the dynamic model for induction machine based on the rotating reference frame using the rotor $\mathrm{d}-\mathrm{q}$ model, which will be described in Section 2. Secondly, in order to obtain the control effects that cannot be achieved by the model reference adaptive control schemes, a fuzzy neural network control (FNNC) with a fast varying-scale optimal method (MDFP) and a fuzzy neural network identifier (FNNI) are employed for the IM speed loop to further enhance the robustness of the system, which will presented in Section 3. Finally, in Section 4, simulations are performed and the results show that the control strategy is feasible, appropriate and effective.

\section{DYNAMIC MODEL OF INDUCTION MACHINES}

According to the theory of induction motor and coordinate transformation method, the rotor field oriented control motion reference model in $d-q$ coordinate reference frame can be given by

$$
\begin{aligned}
\frac{d i_{d 1}}{d t}=- & \left(\frac{L_{m}^{2} R_{2}+L_{2}^{2} R_{1}}{\sigma L_{1} L_{2}^{2}}\right) i_{d 1}+\left(P \omega+\frac{L_{m} R_{2}}{L_{2} \psi_{2}} i_{q 1}\right) i_{q 1} \\
& +\frac{L_{m} R_{2}}{\sigma L_{1} L_{2}^{2}} \psi_{2}+\frac{u_{d 1}}{\sigma L_{1}} \\
\frac{d i_{q 1}}{d t}=- & \left(P \omega+\frac{L_{m} R_{2}}{L_{2} \psi_{2}} i_{q 1}\right) i_{d 1}-\left(\frac{L_{m}^{2} R_{2}+L_{2}^{2} R_{1}}{\sigma L_{1} L_{2}^{2}}\right) i_{q 1} \\
& -\frac{P L_{m} \omega \psi_{2}}{\sigma L_{1} L_{2}} \psi_{2}+\frac{u_{q 1}}{\sigma L_{1}} \\
\frac{d \psi_{2}}{d t}= & \frac{L_{m} R_{2}}{L_{2}} i_{q 1}-\frac{R_{2}}{L_{2}} \psi_{2} \\
\frac{d \omega}{d t}= & P \frac{L_{m}}{J L_{2}} i_{q 1} \psi_{2}-\frac{T_{L}}{J}
\end{aligned}
$$

where $R_{1}$ and $L_{1}$ are the resistance, self-inductance of stator winding respectively; $R_{2}$ and $L_{2}$ the resistance, self-inductance 
of rotor winding; $L_{m}$ the mutual inductance of stator winding and rotor winding; $\psi_{2}$ the flux linkage of rotor winding; $\omega$ the rotor mechanical angular speed; $J$ the rotor mechanism inertia; $T_{L}$ the mechanism torque; $i_{d 1}, i_{q 1}, u_{d 1}$, and $u_{q 1}$ are instantaneous values for current and voltage; $p$ is the time derivative; $q$ for q-axis components; and $d$ for d-axis components.

In a induction machine adjustable speed control system, it is desirable to represent the state variables by a state vector $x$, where

$$
x=\left[i_{d 1}, i_{q 1}, \psi_{2}, \omega\right]^{T}
$$

The control variables for the induction machine are represented by a control vector $u$, where

$$
u=\left[u_{d 1}, u_{q 1}\right]^{T}
$$

The outputs of induction motor are represented by an output vector $y$, where

$$
y=\left[\psi_{2}, \omega\right]^{T}
$$

\section{FUZZY NEURAL NETWORK-BASED ADAPTIVE CONTROL STRATEGY OF INDUCTION MACHINES}

The structure of the designed fuzzy neural network adaptive adjustable speed control system is shown in Fig. 1, which consists of two types of neural network. The fuzzy basis function neural network controller (FNNC) acts as the fuzzy adaptive controller; the fuzzy neural network identifier (FNNI) acts as the model identification of induction motor plant and the back transfer signal $d y_{k} / d u_{k}$, which is used to regulate the network weights $w_{j}$ and parameters $a_{j}, b_{j}$. FNNC and FNNI can be firstly trained off-line according to prior knowledge, and then made learning on-line when the system is running in practice. In addition, a fast varying metric optimal learning algorithm, e.g. the MDFP, is employed to train and correct $w_{j}$, $a_{j}$, and $b_{j}$. In Fig. $1, \mathrm{RM}$ denotes the reference model and IM represents the induction motor.

\section{A. Design of fuzzy neural network controller for IM}

The structure of fuzzy neural network controller shows in Fig.2. The layers (I) to (U) are corresponding to the premise part "IF-part" in the fuzzy control rules; the layer (П) corresponds to the fuzzy inference, which output nodes indicate triggering strength; the layer (IV) corresponds to the conclusion part "THEN-part". The symbol " $\prod$ " denotes the fuzzy AND operating. The symbol "* $*$ denotes the fuzzy minimum operating $[6,7,8]$.

The mapping relation of the inputs and outputs in the FNNC is as follows

The output nodes of the layer (I) are $O_{i}^{(1)}=x_{i}(i=1,2)$

The input and output nodes of the layer (II) are

$$
\begin{aligned}
& I_{i k}^{(2)}=-\left(x_{i}-a_{i k}\right)^{2} / b_{i k}^{2} \quad(k=1,2, \cdots, 7) \\
& O_{i k}^{(2)}=\mu_{A_{i}}\left(x_{i}\right)=\exp \left(I_{i k}^{(2)}\right)
\end{aligned}
$$

The input and output nodes of the layer (III) are

$$
\begin{aligned}
& I_{k}^{(3)}=\prod_{i=1}^{2} O_{i k}^{(2)}=\mu_{A_{1}}\left(x_{1}\right) * \mu_{A_{2}}\left(x_{2}\right) \\
& O_{l}^{(3)}=I_{l}^{(3)} \quad\left(l=1,2, \cdots, k^{2}\right)
\end{aligned}
$$

The input and output nodes of the layer (IV) are

$$
\begin{aligned}
& I_{j}^{(4)}=\sum_{l=1}^{k^{2}} O_{j l}^{(3)} \cdot W_{j l} \quad(j=1,2) \\
& O_{j}^{(4)}=u_{j}=\frac{I_{j}^{(4)}}{\sum_{l=1}^{k^{2}} O_{j l}^{(3)}}
\end{aligned}
$$

where $x_{i}$ denotes the input of FNNC; $w_{j l}$ the weights of the neural network; $a_{i k}$ and $b_{i k}$ the central and width parameters of Gaussian basis function; $u_{j}$ the output control of FNNC.

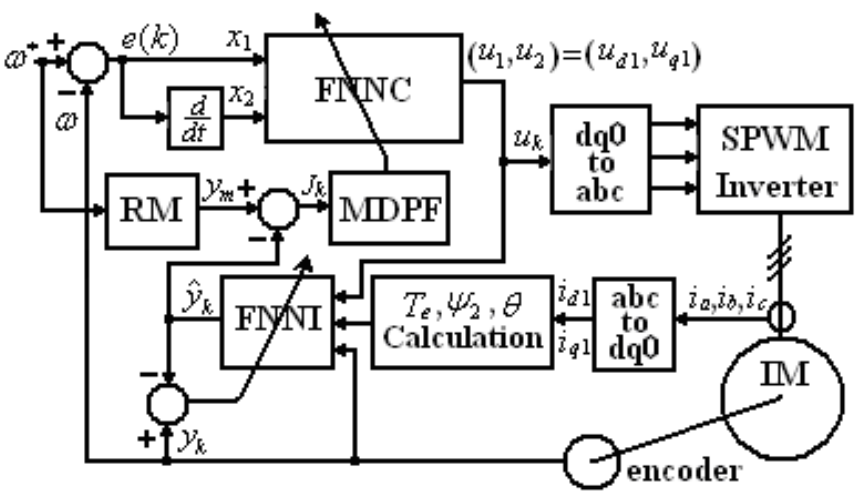

Figure 1. Block diagram for induction machine adjustable speed control system with fuzzy neural network adaptive control strategy

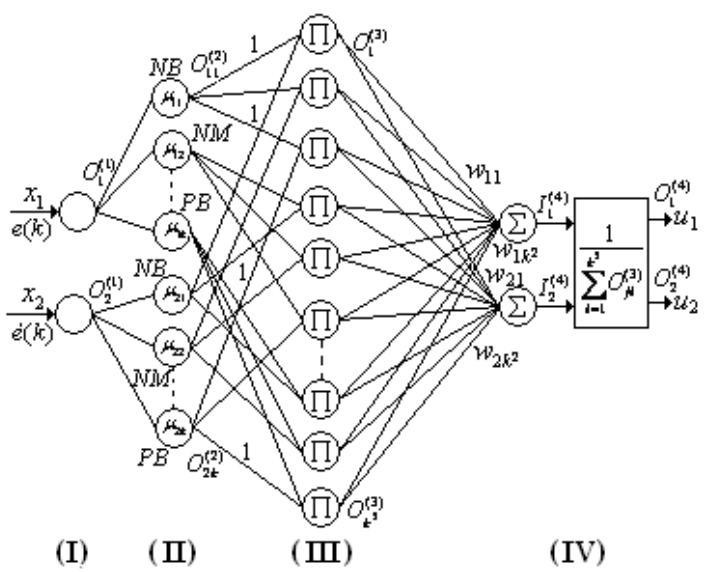

Figure 2. Structure of fuzzy neural network controller for induction machine

\section{B. Design of fast varying metric optimal learning algorithm}

In general, the on-line learning requests that the convergence rate of the learning algorithm is fast, and the stability is well, however, the conventional learning algorithm of neural network, such as the back-propagation (BP) algorithm, is that the convergence rate of the learning algorithm is slower, and the learning rate $\eta$ is uneasy to select, only cut and try, 
which makes the stability worse when the initial weights are trained. The training weights only are local optimum. In this paper, based on the varying metric method (DFP) optimal learning algorithm (MDFP), an improved-type learning algorithm is employed to solve above problems.

The performance index of the error function learning online is defined as

$$
J(W)=\frac{1}{2} \sum_{i=1}^{m} E_{i}^{2}(W)=\frac{1}{2} \sum_{i=1}^{m}\left(y_{m}-y_{k}\right)^{2}
$$

where $W$ is the weights vector of FNNC, $W \in R^{n} ; E_{i}$ denotes the error between the practice system output $y_{k}$ and desired output.

The basic thought of MDFP algorithm is that in the minimum point nearby, the target function $J(W)$ is approximated using the second-order Taylor polynomial formula, and then the estimated value in the minimum point is obtained. The concrete deduced process will be omitted in this paper. The MDFP learning algorithm is written as

$$
\left.\begin{array}{l}
W_{k+1}=W_{k}-\frac{1}{\beta_{k}} H_{k} \cdot E\left(W_{k}\right) \cdot \nabla E\left(W_{k}\right) \\
H_{k+1}=\lambda^{-1}\left(H_{k}-\frac{H_{k} \nabla E\left(W_{k}\right) \cdot \nabla E^{T}\left(W_{k}\right) \cdot H_{k}}{\beta_{k}}\right) \\
\beta_{k}=\lambda+\nabla E^{T}\left(W_{k}\right) \cdot H_{k} \nabla E\left(W_{k}\right) \\
H_{1}=I(\text { Unit Matrix })
\end{array}\right\}
$$

where $\nabla E\left(W_{k}\right)$ is the gradient function of $E$ with respect of $W_{k}$, $0<\lambda<1$.

Because $H_{k}$ is positive definite, according to above formula, $H_{k+1}$ is also positive definite. As $\beta_{k}$ is always positive number, the modified formula $W_{k+1}$ is always kept to be convergence along the negative gradient direction, which ensures the convergence property for the MDFP algorithm.

Therefore, the on-line learning algorithm for FNNC is given by

$$
\left.\begin{array}{l}
a_{k+1}=a_{k}-H_{k} E_{k}\left(a_{i k}\right) \cdot \nabla E_{k}\left(a_{i k}\right) / \beta_{k} \\
b_{k+1}=b_{k}-H_{k} E_{k}\left(b_{i k}\right) \cdot \nabla E_{k}\left(b_{i k}\right) / \beta_{k} \\
W_{k+1}=W_{k}-H_{k} E_{k}\left(W_{k}\right) \cdot \nabla E_{k}\left(W_{k}\right) / \beta_{k} \\
H_{k+1}=\lambda^{-1}\left(H_{k}-H_{k} \nabla E_{k} \cdot \nabla E_{k}^{T} \cdot H_{k} / \beta_{k}\right) \\
\beta_{k+1}=\lambda+\nabla E_{k}^{T} \cdot H_{k} \cdot \nabla E_{k}
\end{array}\right\}
$$

where $E_{k}=y_{m}-y_{k}, H_{1}=I, \nabla E_{k}$ indicates the gradient of $\nabla E_{k}\left(a_{i k}\right), \nabla E_{k}\left(b_{i k}\right)$, and $\nabla E_{k}\left(W_{k}\right)$ respectively, which are determined by

$$
\nabla E_{k}\left(W_{k}\right)=-E_{k}\left(\frac{O_{k}^{(3)}}{\sum_{k=1}^{m} O_{k}^{(3)}}\right) \cdot\left(\frac{\partial y_{k}}{\partial u_{k}}\right)
$$

$$
\begin{array}{r}
\nabla E_{k}\left(a_{i k}\right)=-2 E_{k}\left[W_{k} \sum_{j=1}^{m} O_{j}^{(3)}-\sum_{j=1}^{m}\left(O_{j}^{(3)} \cdot W_{j}\right)\right] \\
\times\left(x_{i}-a_{i k}\right) \cdot O_{k}^{(3)} /\left[b_{i k}^{2}\left(\sum_{j=1}^{m} O_{j}^{(3)}\right)^{2}\right] \times\left(\frac{\partial y_{k}}{\partial u_{k}}\right) \\
\nabla E_{k}\left(b_{i k}\right)=-2 E_{k}\left[W_{k} \sum_{j=1}^{m} O_{j}^{(3)}-\sum_{j=1}^{m}\left(O_{j}^{(3)} \cdot W_{j}\right)\right] \\
\times\left(x_{i}-b_{i k}\right)^{2} \cdot O_{k}^{(3)} /\left[b_{i k}^{3}\left(\sum_{j=1}^{m} O_{j}^{(3)}\right)^{2}\right] \times\left(\frac{\partial y_{k}}{\partial u_{k}}\right)
\end{array}
$$

The partial derivative $\left(\partial y_{k} / \partial u_{k}\right)$ mentioned above can not be obtained directly in the condition of unknown model of the plant. Hence, a fuzzy neural network identifier (FNNI) can be gotten according to the back propagation.

\section{Design of fuzzy neural network identifier}

In order to dynamically estimate the model of unknown plant, meanwhile, the partial derivative $\left(\partial y_{k} / \partial u_{k}\right)$ can be provided for FNNC, based on a three layers BP neural network, a FNNI is designed to realize the modeling of system.

Suppose that the relationship between the input and output in the identified system is as follows

$$
\begin{aligned}
y_{k}= & f\left(y(k-1), \cdots, y\left(k-n_{y}\right), u(k-1),\right. \\
& \left.\cdots, u\left(k-n_{u}\right)\right)+n(k)
\end{aligned}
$$

where $y(k)$ and $u(k)$ denote the output and input variables of system respectively; $f(\bullet)$ is a nonlinear function; $n_{y}$ and $n_{u}$ are the time delay of the output and input variables; $n(k)$ is noisy.

The neural network model of identification system is described by

$$
\begin{aligned}
\hat{y}_{k} & =f(x(k), W)=\sum_{i=1}^{n_{h}} W_{k i}^{(0)} \cdot O_{i}^{h}(k) \\
& =\sum_{i=1}^{n_{h}} W_{k i}^{(0)} \bullet F\left(\sum_{j=1}^{n} W_{i j}^{(h)} \cdot x_{j}(k)-\theta_{i}^{(h)}\right) \quad(1 \leq k \leq m)
\end{aligned}
$$

Where $W_{k i}^{(0)}$ and $W_{i j}^{(0)}$ denote the weights of hidden layer and output layer respectively; $\theta_{i}^{(h)}$ is the threshold value of hidden layer; $x(k)$ represents the total variables of the identified system, which takes the form

$$
\begin{aligned}
x(k) & =\left[x_{1}(k), x_{2}(k), \cdots, x_{n}(k)\right]^{T} \\
& =\left[y(k-1), \cdots, y\left(k-n_{y}\right), u(k-1), \cdots, u\left(k-n_{u}\right)\right]^{T}
\end{aligned}
$$

If it is known that there are $\mathrm{N}$ groups of input and output samples $\{u(k), y(k)\}(k=1,2, \cdots, N)$, the determining problem of the parameters $\left(W^{(0)}, W^{(h)}, \theta^{(h)}\right)$ for FNNI will be transformed into optimal problem of below function, which is defined as

$$
J^{M}(W)=\frac{1}{2} \sum_{k=1}^{N}\left[E_{k}^{M}(W)\right]=\frac{1}{2} \sum_{k=1}^{N}\left(y_{k}-\hat{y}_{k}\right)^{2}
$$




\section{SIMULATION RESULTS}

In order to evaluate the correctness and feasibility of the proposed control strategy, the characteristics of a three-phase induction machine, as shown in Fig. 1, has been simulated using MATLAB/SIMULINK. The parameters of IM are

$$
\begin{aligned}
& P_{\text {nот }}=1.75 \mathrm{~kW}, \quad N_{p}=2 \quad, \quad n_{\text {nom }}=1441 \mathrm{r} / \mathrm{min}, \\
& T_{\text {enom }}=8.84 \mathrm{~N} \cdot \mathrm{m}, \quad I_{\text {nот }}=2.6 \mathrm{~A}, \quad r_{1}=4.25 \Omega, \\
& r_{2}=3.24 \Omega, L_{1}=0.666 \mathrm{H}, L_{2}=0.671 \mathrm{H}, L_{m}=0.651 \mathrm{H}, \\
& J=0.02 \mathrm{~N} \cdot \mathrm{m}^{2} .
\end{aligned}
$$

In the process of simulation, we use 49 fuzzy control rules as the off-line learning samples $\left(x_{1}, x_{2} \rightarrow u_{1}, u_{2}\right)$. The structure of FNNC is 2-14-49-2, where the central values $a_{i}(0)(i=1,2, \cdots, 7)$ of Gaussian membership function respectively take the form $\{\mathrm{NB}, \mathrm{NM}, \mathrm{NS}, \mathrm{ZO}, \mathrm{PS}, \mathrm{PM}, \mathrm{PB}\}$; The initial width $b_{j}(0)=2.5^{2}(j=1,2, \cdots, 7)$, which belongs to in the universe[-6, 6].

The simulation results of designed FNNC based on a FNNI show in Fig. 3, 4, and 5. Fig. 3 shows the starting characteristic with the proposed control strategy when the IM can operate from 0 to $1500 \mathrm{rpm}$ and the torque $T_{e}$ quickly arrive the limited value, and exit the saturation after $0.12 \mathrm{~s}$, as shown in Fig. 4, the system enters the stable state. When the time is $0.3 \mathrm{~s}$, meanwhile, the speed changes from $1500 \mathrm{rpm}$ to $1200 \mathrm{rpm}$, and when time is $0.33 \mathrm{~s}$, the adjustable speed system can reach new static speed, as shown in Fig. 3. The current responding curve is illustrated in Fig. 5 during the operating process.

Because the FNNC is that the fuzzy neural network control combines with the conventional the MRAC, the IM adjustable speed system integrates the advantages between them. In other words, the system can keep the characteristics of FNNC and MRAC, which can realize the dynamic decoupling control for the torque and magnetic flux of IM. Therefore, the proposed system is of excellent speed responding performance, robustness, and strong anti-interference ability, such as fast speed responding, no speed overshoot, no static speed error, and so on.

\section{CONCLUSION}

Based on a fast varying metric method (MDFP) and a fuzzy neural network identifier (FNNI), a fuzzy neural network

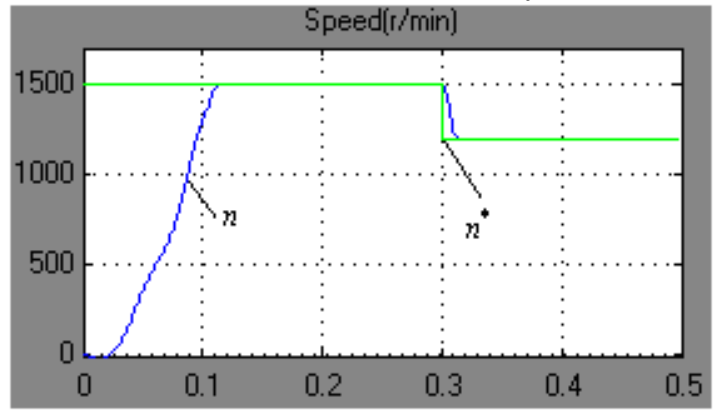

Figure 3. Step responding starting characteristics

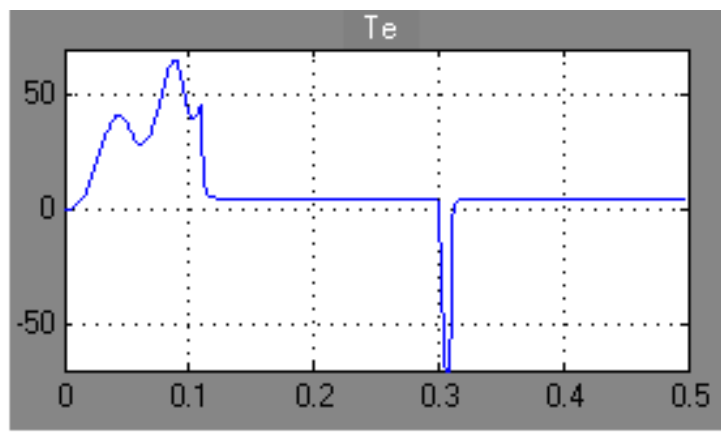

Figure 4. Step change of load torque

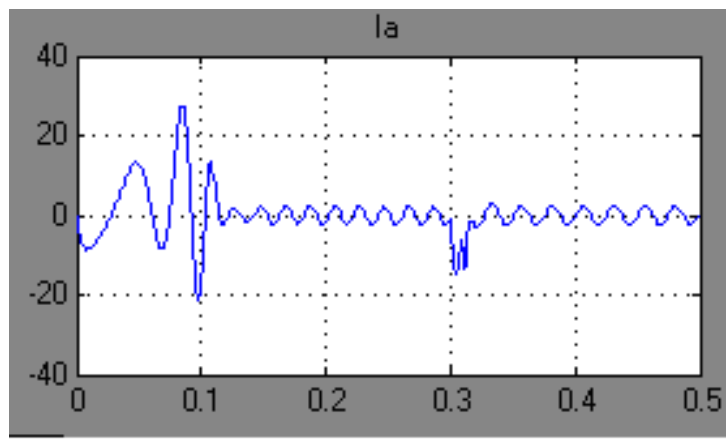

Figure 5. Current responding curve when load changes

adaptive controller (FNNC) for the three-phase induction machine adjustable speed system has been implemented. The proposed FNNC controller is not only of the adaptive learning function, but also can realize the fuzzy control. The designed processes of FNNC, MDFP, and FNNI algorithms have been described in details. Simulation results show the feasibility, correctness and effectiveness of the proposed control strategy, such as the excellent static and dynamic performances, and strong anti-interference ability.

\section{REFERENCES}

[1] X. M. Deng, Electromagnetic analysis and design applications of Brushless Doubly Fed Machine, China Machine Press, January 2009.

[2] G. H. Liu, Z. J. Hu, Y. Shen, H. W. Zhou, and C. L. Teng, "Estimation of Induction Motor Speed Based on Artificial Neural Networks Inversion System,” EEE Int. Conference Neural Networks \& Signal Processing, Zhenjiang, China, June 2008.

[3] Z. F. Li, G. Zhang, L. J. Diao, and Z. G. Liu, "Extended Kalman Filter Based on Inverse \& Model of Induction Motor," IEEE Vehicle Power and Propulsion Conference (VPPC), Harbin, China, September 2008.

[4] X. X. Liu, L. Dong, W. J. Fan, and Y. X. Sun, "Decoupling Control of the 5 Degree-of-Freedom Bearingless Induction Motor Based on a -th Order Inverse System Method,” Proc. of the 26th Chinese Control Conference, Zhangjiajie, Hunan, China, July 2007.

[5] X. Z. Dai, and X. Wang, "Neural Network Inverse Control of CurrentFed Induction Motor,"

[6] Y. N. Wang. Intelligent Control System. Chian Hunan University Press, 2006.

[7] Y. Wang, "A neural network adaptive control based on rapid learning method and its application," Advances In Modeling and Analysis, Vol. 46(3), pp. 27 34, 1994.

[8] C. T. Lin, "Neural-network-based Fuzzy Logic Control and Decision System,” IEEE trans. on Computers, Vol. 40(12), pp. 1320-1336, 1991. 